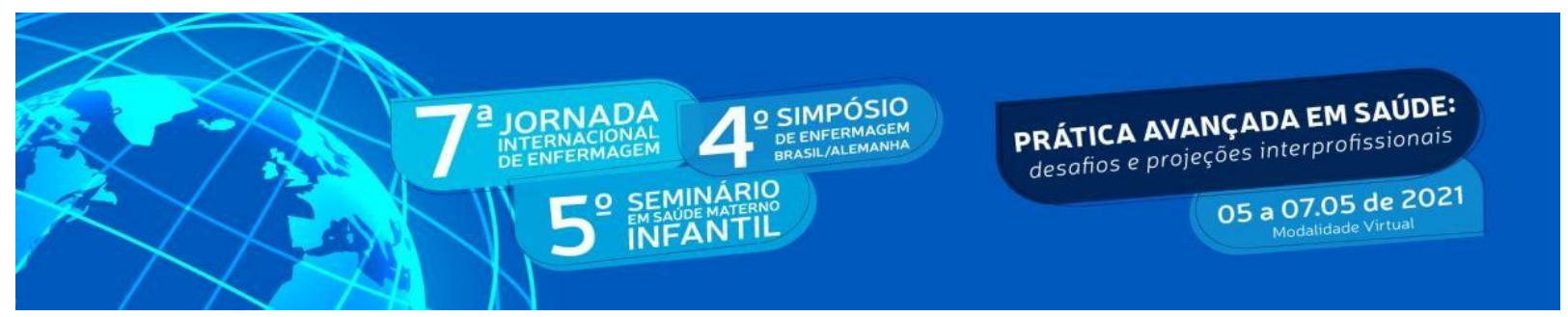

\title{
ABANDONO VACINAL EM CRIANÇAS NO ANO DA PANDEMIA EM MINAS GERAIS
}

\section{Gabriela Lourença Martins do Nascimento ${ }^{1}$; Rayssa Nogueira Rodrigues²; Marialice Caetano da Silva ${ }^{3}$; Gabriela Cunha Corrêa Freitas de Oliveira ${ }^{4}$; Ana Clara Morais Amaral $^{5}$; Eliete Albano de Azevedo Guimarães ${ }^{6}$}

\begin{abstract}
RESUMO
Descrever o comportamento espaço-temporal da proporção de abandono na vacinação de rotina de crianças menores de um ano no estado de Minas Gerais. Trata-se de um estudo ecológico, descritivo. Os dados foram obtidos por meio do Departamento de Atenção Básica de Secretaria de Atenção à Saúde, do Ministério da Saúde. A proporção de abandono de vacinas com esquema multidoses foi avaliado por macrorregiões entre os meses de janeiro a dezembro de 2020. O Software Microsoft Excel foi utilizado para o tratamento e análise estatística. O estudo apresentou heterogeneidade nas proporções de abandono das vacinas rotavírus, pneumocócica 10 valente, pentavalente, tendo picos nos meses julho, fevereiro, janeiro e março, respectivamente. Já a poliomielite não apresentou predominância de abandono entre os meses estudados, sendo verificadas grandes oscilações para a mesma.
\end{abstract}

Palavras-chave: Criança; Medidas em Epidemiologia; Vacinas.

\begin{abstract}
ABSCTRACT
Describe the space-time behavior of the proportion of abandonment in the vaccination routine of children under 1 year of age in the State of Minas Gerais. It is about ecological, descriptive study. The data were obtained through The Basic Care and Attention Department of the Health Care, from the Brazilian's Ministry of Health. The proportion of abandonment of vaccines with a multi-dose scheme was assessed by macro-regions between the months of January to December 2020. The Excel Microsoft Software was used for the treatment and statistical analysis. The study showed heterogeneity in the proportions of abandonment rates in following vaccines: rotavirus, pneumococcal-10-valiant, pentavalent vaccines, with peaks in July, February, January and March, respectively. On the other hand, Polio did not show a predominance of abandonment among the months studied, with large fluctuations for the same.
\end{abstract}

Key-words: Child; Measures in Epidemology; Vaccines.

\footnotetext{
${ }^{1}$ Estudante do curso de enfermagem da Universidade Federal de São João Del Rei (UFSJ). E-mail: gabimartinsenf@gmail.com

${ }_{2}^{2}$ Pós-doutoranda em saúde coletiva pela Universidade Federal de São João Del Rei (UFSJ). Professora da Universidade Federal de Viçosa (UFV). E-mail: rayssa.machado@ufv.br

${ }^{3}$ Estudante do curso de enfermagem da Universidade Federal de São João Del Rei (UFSJ). E-mail: marialicecs@aluno.ufsj.edu.br

${ }^{4}$ Mestranda em ciências da saúde com ênfase em Enfermagem pela Universidade Federal de São João Del Rei (UFSJ). E-mail: gabyccunha@gmail.com

5 Estudante do curso de enfermagem da Universidade Federal de São João Del Rei (UFSJ). E-mail: anaclaramoraisa2@aluno.ufsj.edu.br

${ }^{6}$ Orientador. Pós-doutora em saúde coletiva. Professora da Universidade Federal de São João Del Rei (UFSJ). Email: elietealbano@ufsj.edu.br
} 


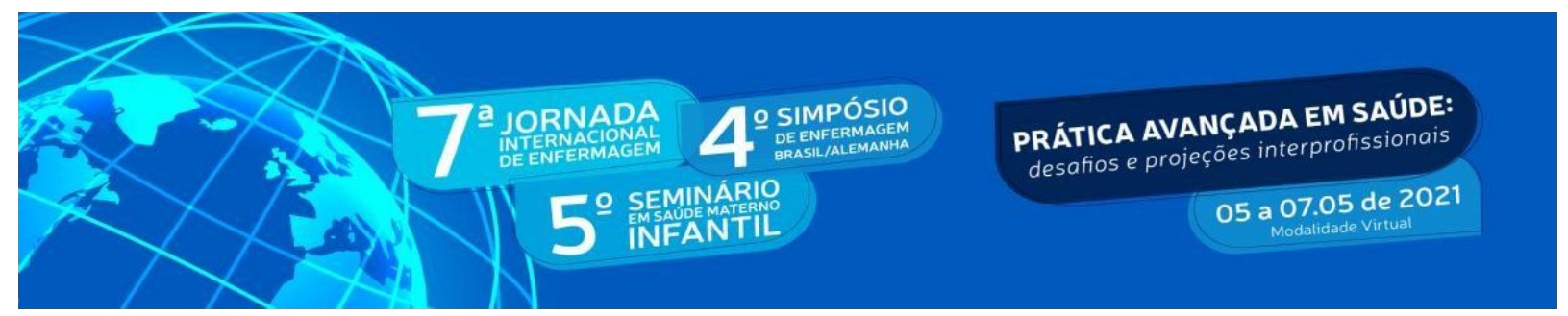

\section{INTRODUÇÃO}

As ações de imunização têm propiciado a eliminação e o controle de várias doenças imunopreveníveis, além de obter um custo-benefício de grande relevância (FERREIRA, et al 2018). No Brasil, desde o ano 1973 existe o Programa Nacional de Imunização (PNI), com o objetivo de oferecer 19 vacinas que contemplam todos os públicos alvos (crianças, adolescentes, adultos, indígenas, idosos e portadores de casos clínicos especiais) (BRASIL,2020). Durante sua trajetória, o PNI enfrentou diversos desafios dentre estes, destaca-se a hesitação vacinal, que é o atraso em receber as doses das vacinas ou a recusa em adesão da imunização (SATO, 2020).

O impacto mais significativo da não vacinação tem sido a morbidade e mortalidade por infecções, principalmente, em crianças (RODRIGUES; PLOTKIN, 2020). A carga da doença é alta neste estágio inicial devido à natureza única do sistema imunológico. A transição do ambiente protegido no útero para o "mundo externo", a falta de defesa contra anticorpos induzidos pela vacina e o perfil dos primeiros patógenos contribuem para a vulnerabilidade desse público (SASO; KAMPMANN, 2017).

Esse cenário se tornou mais preocupante a partir de 2020. Com a ocorrência da pandemia causada pelo SARS-CoV-2, o novo coronavírus (COVID-19), a demanda de vários serviços de saúde caiu drasticamente, incluindo a vacinação (SATO, 2020). Inclusive, no início da pandemia a Organização Pan Americana de Saúde (OPAS) recomendou que as campanhas vacinais fossem paralisadas e que fosse feito somente as vacinas de rotina, a fim de evitar a retomada de doenças imunopreveníveis (OPAS, 2020).

Nesse sentido, a provável queda das coberturas vacinais, em especial, as infantis e suas consequências justificam os esforços para propor um planejamento estratégico condizente com as características de cada localidade. Assim, o presente estudo propôs conhecer a proporção de abandono de vacinas em crianças menores de um ano nas macrorregiões pertencentes ao estado de Minas Gerais no ano de 2020, segundo mais populoso do Brasil e o quarto em extensão territorial (IBGE, 2020).

\section{OBJETIVO}

Descrever o comportamento espaço-temporal da proporção de abandono na vacinação de rotinas de crianças menores de um ano no estado de Minas Gerais. 


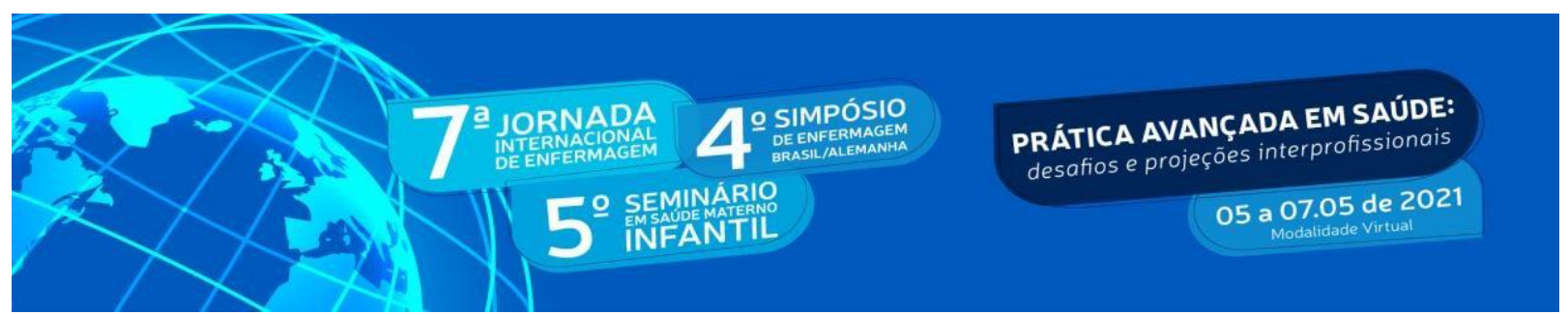

Gráfico 4- Proporção de abandono vacinal por macrorregião para vacina poliomielite dos meses referentes ao ano de 2020 no estado de Minas Gerais

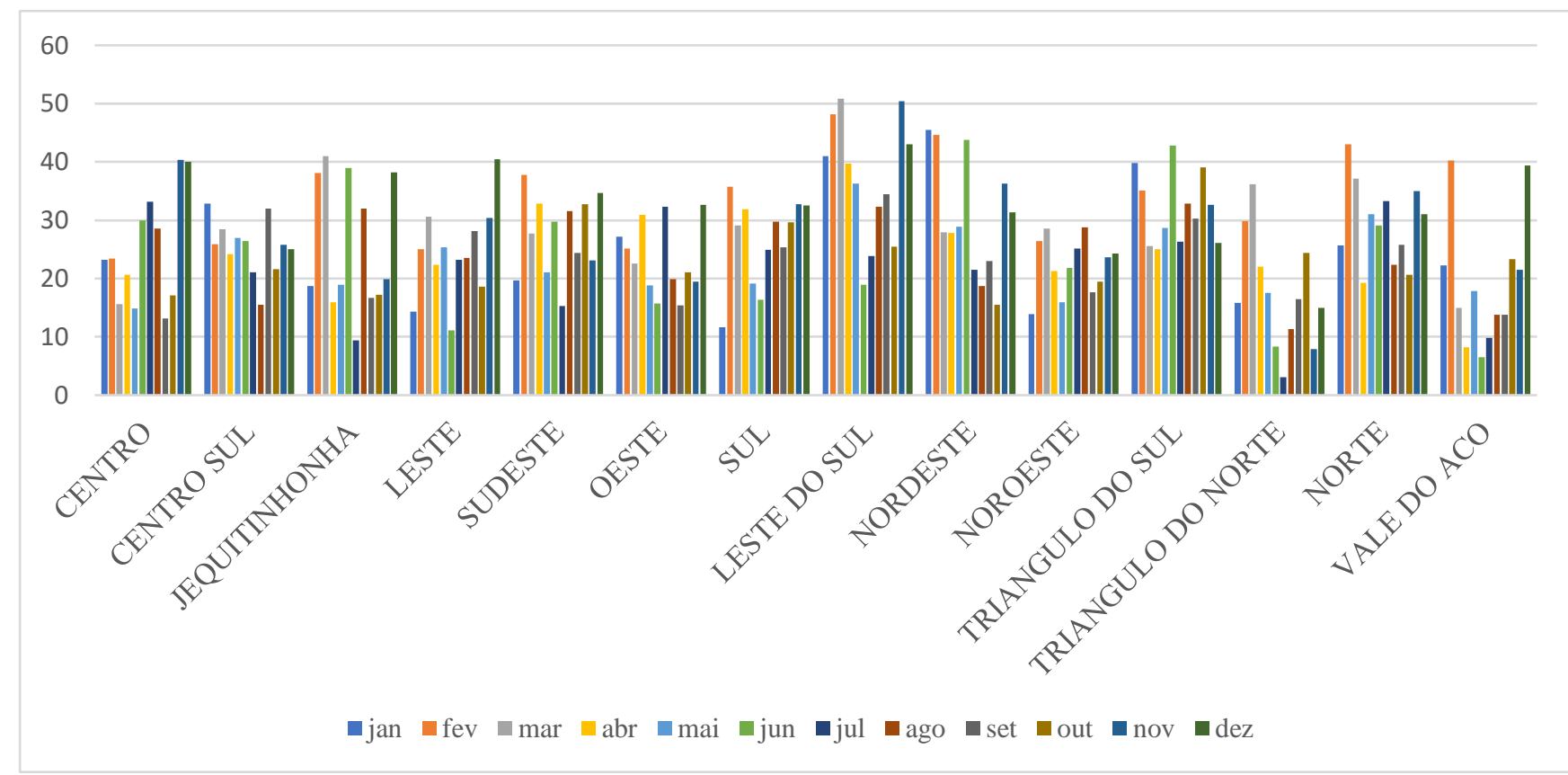

Fonte: dados do DAB/SAS/MS

Diante desse cenário, ações de educação em saúde são necessárias, a fỉm de reforçar a importância da vacinação. Estudo de risco-benefício em países africanos mostrou que as mortes evitáveis pela vacinação de rotina superam o excesso de risco de morte pela COVID-19 associado ao comparecimento no serviço de saúde para a vacinação, demonstrando a importância de esforços voltados a aumentar as coberturas vacinais neste momento (ABBAS et al., 2020; SUWANTIKA et al., 2020).

Outro ponto que deve ser destacado é a estrutura do sistema público de saúde no Brasil, especialmente a Estratégia Saúde da Família (ESF). A incorporação de um novo membro ao processo de trabalho: o agente comunitário de saúde (ACS), contribui para vigilância da situação de saúde das famílias de sua área adscrita. Nesse sentido, pode-se pensar que a redução/suspensão das visitas domiciliares realizada por esse profissional, devido a pandemia, pode ter contribuído para o aumento do abandono vacinal (ABBAS et al., 2020; SUWANTIKA et al., 2020).

Por fim, merece destaque que essa situação gera riscos de saúde públicos de proporções imensuráveis diante do retorno às aulas. Dados apontam que o retorno às aulas poderá ampliar o risco não só de expansão das epidemias de sarampo em todo o país, mas também de 


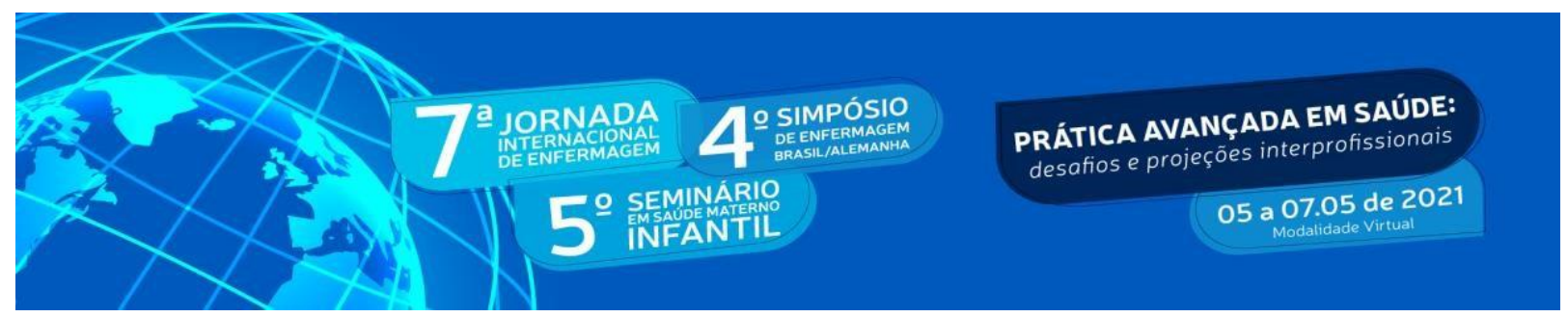

reemergência de outras doenças já controladas, das quais vale salientar a difteria e a coqueluche (SATO,2020).

Sabendo que os problemas citados são intensificados no contexto da pandemia pode-se incentivar os profissionais e usuários quanto a adesão vacinal, por meio do agendamento para atendimento vacinal de forma a garantir segurança ao paciente, estimulação da vacinação de rotina, compartilhamento de informações seguras acerca da vacinação, organização da gestão e administração dos imunobiológicos de modo acessível e facilitado, como por exemplo, pontos drive thru. Objetivando assim maior adesão da vacinação e da continuidade do esquema vacinal, sendo a vacinação um serviço de saúde essencial, que deve ter prioridade na prevenção das doenças transmissíveis e ser protegido para continuidade durante a pandemia de COVID19, contribuindo para a saúde social e individual.

Entre as limitações de estudos dessa natureza cabe destacar que a utilização de dados secundários pode frequentemente resultar em inconsistências nos indicadores estimados, mas, apesar disso, a escolha por esse tipo de fonte reduz os custos operacionais e não inviabiliza a realização de análises. Para estudos futuros seria oportuno a análise com modelos estatísticos, a fim de identificar a significância estatísticas dos dados encontrados.

\section{CONCLUSÃO}

Apesar da heterogeneidade nas taxas de abandono das vacinas VORH, pneumocócica 10 valente, pentavalente e poliomielite nas diversas macrorregiões do estado de Minas Gerais é notável que as vacinas continuam a ser uma das ferramentas mais eficazes para prevenir a morbidade e mortalidade de ameaças endêmicas e emergentes. Avanços recentes anunciam uma era inovadora das vacinas. No entanto, a desinformação, as fake News, o negacionismo, os movimentos antivacinismo, a pandemia COVID - 19 e a consequente desconfiança nas vacinas representam uma ameaça ao seu sucesso e impacto positivo na saúde humana global. Neste contexto podemos relacionar as oscilações na taxa de abandono vacinal nas macrorregiões de Minas Gerais, a esses processos, o que nos faz pensar em estratégias mais do que nunca imprescindíveis para informar a população, comunicar a verdade científica educando assim o 


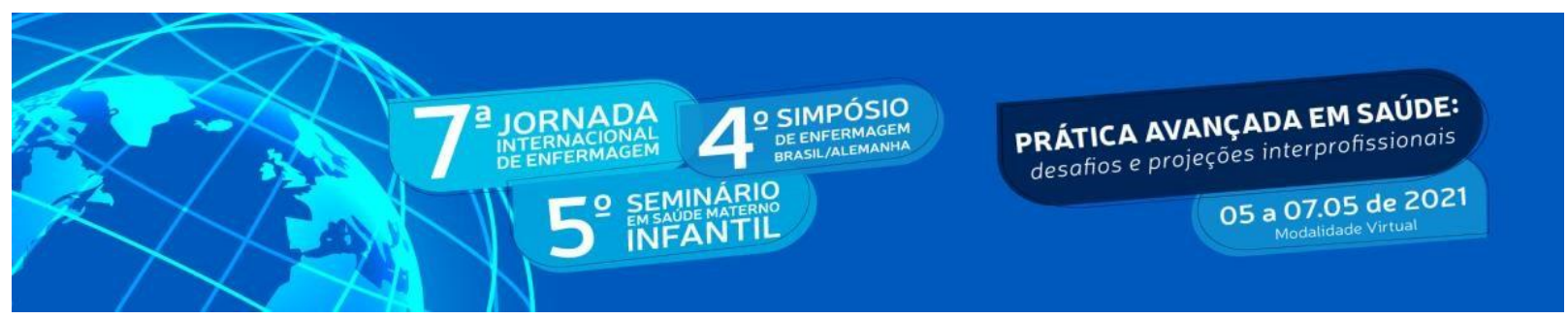

público sobre a segurança e os benefícios da vacinação, usando as mídias tradicionais e sociais, além de criar estratégias para atingir o público alvo que está receoso a se deslocar a unidade devido as medidas de distanciamento propostas pelas organizações de saúde afim de paralisar a pandemia de COVID-19.

Portanto, sugere-se que maiores estudos sejam feitos referentes à proporção de abandono vacinal no contexto regional e nacional, além de divulgar no futuro a ciência das vacinas não apenas em disciplinas como virologia, imunologia, bioinformática e biologia de sistemas, mas também nas ciências sociais tornando o assunto uma pauta rotineira entre a sociedade e o serviço. Gerando assim a promoção da saúde e prevenção de agravos e doenças um trabalho multidisciplinar entre os prestadores de cuidados de saúde, autoridades de saúde pública nos níveis local, estadual e federal e população para dessa forma atingir os níveis desejados de vacinação, continuando o processo de controle, erradicação e eliminação das doenças imunopreviníveis.

\section{REFERÊNCIAS}

ABBAS, $\mathrm{K}$ et al. Routine childhood immunisation during the COVID-19 pandemic in Africa: a benefit-risk analysis of health benefits versus excess risk of SARS-CoV-2 infection. The Lancet Global Health, v. 8, n. 10, p. e1264-e1272, out. 2020.

BRASIL. Ministério da Saúde. Conselho Nacional de Saúde. Comissão Nacional de Ética em Pesquisa. Resolução no 466, de 12 de dezembro de 2012. Diário Oficial da União: seção 1, Brasília, DF, n. 112, p. 59-62, 13 jul. 2013.

BRASIL. Ministério da Saúde. Calendário Nacional de Vacinação. 2020. Disponível em: https://www.saude.go.gov.br/files/imunizacao/calendario/Calendario.Nacional.Vacinacao.202 0.atualizado.pdf calendario 2020.

BRASIL. Domingues, C.M.A.S. Ciclos de estudos CGDEP. 2015. Disponível em: https://portalarquivos.saude.gov.br/images/pdf/2015/novembro/09/Ciclo-de-estudos-CGDEP2015-Final-CarlaMagda.pdf. Acesso em fev de 2021

DATASUS. SI-PNI - Sistema de Informações do Programa Nacional de Imunizações. Disponível em: http://tabnet.datasus.gov.br/cgi/pni/cpnidescr.htm\#: :text=A\%20f\%C3\%B3rmula\%20de\%20c \%C3\%A1lculo\%20da,de\%20menores\%20de\%201\%20ano. Acesso em 13 de mar.2021. 
